\title{
'Hot' new treatment for HIV
}

Radioactive antibodies can seek out and kill HIV-infected blood cells in mouse models. If the same proves true in humans, radioimmunotherapy may provide a new way to combat HIV infection by eliminating viral reservoirs.

The AIDS epidemic is now 25 years old, but there is still no cure for the disease. The treatment of HIV was revolutionized in the late 1990s with the advent of highly active antiretroviral therapy (HAART), drug combinations that target and block HIV enzymes (such as reverse transcriptase inhibitors and protease inhibitors), thus preventing viral replication. HAART has helped to slow disease progression and prolong the lives of many infected with HIV, but also has serious side effects. Moreover, the emergence of drug-resistant HIV strains threatens the continued effectiveness of drug-cocktail treatments like HAART. In addition, although HAART may reduce circulating HIV to undetectable levels, it cannot cure the disease because latently infected cells harbor replication-competent virus.

Now, in new research based on the use of radiolabeled antibodies for treatment of certain cancers, Ekaterina Dadachova of the Albert Einstein College of Medicine (Bronx, NY) and colleagues tested the efficacy of such antibodies in eliminating HIVinfected cells that express viral proteins on their surface. The researchers linked antibodies against the HIV surface glycoproteins gp120 and gp41 with radioisotopes of bismuth $\left({ }^{213} \mathrm{Bi}\right)$ and rhenium $\left({ }^{188} \mathrm{Re}\right)$, then assayed the ability of the radioactive antibodies to kill HIV-infected cells in vitro (PLoS Medicine, November 2006).

Next, Dadachova's team investigated the same technique, called anti-HIV radioimmunotherapy (RIT), in vivo. They injected SCID mice intrasplenically with HIV-infected human peripheral blood mononuclear cells and then treated them with radioactive antibodies. Ninety-nine percent of the HIVinfected cells were eliminated. Likewise, in SCID mice with human thymic graft implants, RIT resulted in a 2.5 -fold decrease in the number of HIV-infected thymocytes. Platelet counts in treated mice suggest that RIT was not associated with acute hematologic toxicity, except at the highest doses of radioactivity.

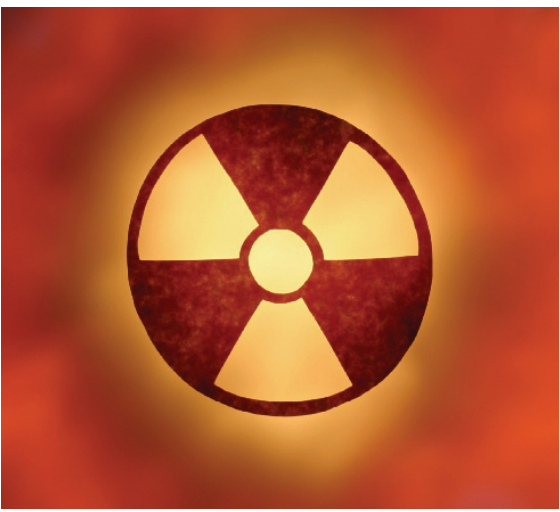

"Radioimmunotherapy in combination with other drugs may form a basis for a cure [for HIV infection] because [RIT] has the potential to find and eliminate 'viral factories' - the infected cells harboring HIV deep inside organs and tissues," Dadachova tells Lab Animal. In addition, RIT may be able to eradicate cells infested with drugresistant virus. Dadachova's team, with an industrial partner, has begun preparation for clinical trials of the therapy, which may begin in less than two years.

Tanja Schub

\section{A NEW STANDARD FOR RABTES VACCTNES?}

A new paper focusing on the long-standing World Health Organization (WHO)-recommended test for the potency of rabies vaccine indicates that it may not be the best method for testing the vaccine, and that another method would be more effective and use fewer animals.

Rabies has been eradicated in many European countries, and even in those developed countries like the United States where the disease still exists, vigorous immunization campaigns, especially in dogs, have brought the number of rabies-related human deaths down to only a handful each year. Still, the estimated human morbidity in developing countries remains high, killing roughly 50,000 or more annually.

One reason for successful rabies treatment in developed countries is that the vaccine can be effectively administered after exposure to the virus. The WHO recommends that newly manufactured lots of human and veterinary rabies vaccine be tested by an in vivo mouse test called the 'National Institutes of Health rabies vaccine potency test' or 'NIH test.'

Although this test is widely regarded as 'adequate,' it is not ideal. There has been concerted effort, for instance, to develop a replacement test that uses fewer or no animals at all. Beyond this, the NIH test has been criticized for its potential to produce inaccurate vaccine potency ratings because its method of vaccine administration and challenge do not adequately mimic actual immunization practice or natural infection routes.

Now, a study published in the 30 November 2006 issue of Vaccine lends credence to the idea that the NIH test should be re-evaluated and possibly replaced. The study, led by Peter Wunderli at ImmunoGen (Cambridge, MA), evaluated experiments performed in the 1990s comparing the accuracy of the NIH test to that of another measure of vaccine potency, the rabies peripheral challenge (RPC) test. Although the RPC test is also an in vivo test, it has the advantage of more closely mimicking routine immunization and natural infection, and uses fewer mice.

The study found that the RPC test picked up significant differences in potency between sequential lots of manufactured vaccine that were missed by the NIH test. Moreover, the RPC test was able to evaluate the protection offered by recombinant-based subunit vaccines, something the NIH test cannot do. As Wunderli tells Lab Animal, adopting the RPC test would mean "fewer mice, more sensitivity, a simpler testing and approval process for current vaccines, and more cost effective recombinant-based subunit vaccines." To win widespread approval for the RPC test, however, a larger collaborative international study still must be conducted to compare the RPC test to other in vivo testing methods.

Owen Young 